REGARDS

SUR L'ECONOMIE ALLEMANDE

BULLETIN ECONOMIQUE DU CRAC

\section{Regards sur l'économie allemande}

Bulletin économique du CIRAC

98-99 | 2010

Varia

\title{
Compagnons de voyage. Les médias et l'Unité allemande
}

\section{Uwe Kammann}

Traducteur : Isabelle Bourgeois

\section{OpenEdition \\ Journals}

Édition électronique

URL : http://journals.openedition.org/rea/4187

DOI : $10.4000 /$ rea. 4187

ISBN : 978-2-8218-0892-8

ISSN : 1965-0787

Éditeur

CIRAC

Édition imprimée

Date de publication : 26 octobre 2010

Pagination : 47-61

ISSN : 1156-8992

\section{Référence électronique}

Uwe Kammann, « Compagnons de voyage. Les médias et l'Unité allemande », Regards sur l'économie allemande [En ligne], 98-99 | octobre 2010, mis en ligne le 01 octobre 2012, consulté le 01 mai 2019. URL : http://journals.openedition.org/rea/4187 ; DOI : 10.4000/rea.4187 


\section{Compagnons de voyage. Les médias et 1'Unité allemande}

\section{Uwe Kammann}

La représentation ou, plus précisément : la vision que nous avons de l'Unité allemande est essentiellement l'œuvre des médias. Karl Brenke ne rappelle-t-il pas dans ce numéro le rôle fondamental joué par une petite phrase du chancelier Kohl dans la perception de l'état de l'économie est-allemande ? Eugen Spitznagel ne montre-t-il pas, lui aussi, en filigrane, le caractère vivace du rêve perdu des "paysages fleuris » quand on regarde le marché du travail dans les nouveaux Länder? Et Thomas Petersen ne nous retrace-t-il pas à quel point l'évolution de l'opinion allemande au fil des 20 ans écoulés est fonction de la relation que les médias font des événements ?

Nous avons donc demandé à un acteur de l'information, un de ceux qui ont accompagné au jour le jour les événements qui se bousculaient depuis l'été 1989, puis le processus de l'unification jusqu'à aujourd'hui, de nous faire part de son témoignage comme de son analyse. Uwe Kammann exerçait alors de hautes responsabilités : il était rédacteur en chef du service médias (Kirche und Rundfunk, rebaptisé ensuite epd medien) de l'agence d'information de l'Eglise protestante d'Allemagne: epd (créée en 1948. En tant que fournisseur d'information aux autres médias, il était tenu au profond respect de la déontologie, à commencer par la rigueur dans l'exposé des faits ; mais il était aussi observateur et critique des contenus délivrés par les médias : en tant que tel, il lui revenait en parallèle d'examiner et de relater la manière dont les autres présentaient les faits. Revivons avec lui les étapes d'un processus à l'issue duquel l'Allemagne s'est forgé une nouvelle identité... (IB)

«Anschluss »: le ministre-président social-démocrate du Brandebourg, Mathias Platzeck, avait eu ce mot dans les jours précédant la célébration du $20^{\mathrm{e}}$ anniversaire de l'Unité allemande - déclenchant une belle polémique. Richard Schröder, qui présidait la commission du SPD est-allemand de la Volkskammer (le parlement de la RDA) en 1990, exigea par exemple qu'il soit renoncé, même dans les polémiques internes au monde politique, à toute comparaison avec l'époque nazie. Même en 1990, il était "totalement erroné » d'établir une relation entre l'adhésion de la RDA au territoire où s'applique la Loi fondamentale avec « l'Anschluss » de l'Autriche à l'Allemagne nazie en mars 1933. Le secrétaire général fédéral de la CDU, Hermann Gröhe, dit qu'il était «honteux » que M. Platzeck, qui avait été un défenseur des droits du citoyen en RDA, s'approprie ainsi « l'amalgame historique contre nature de ce parti incurablement passéiste qu'est Die $L I N K E$ ». La vérité est autre : la réunification des deux Etats allemands répondait à « la volonté clairement exprimée de presque tous les citoyens de l'ex-RDA ».

Cet échange était un des rares d'une telle force qu'on ait pu relever dans les innombrables positions et réflexions précédant la célébration de l'Unité. En effet, on pouvait observer au contraire que tant les prises de positions des personnalités politiques que les commentaires et analyses de presque tous les médias suivaient une ligne relativement homogène - à la grande surprise de nombre de contemporains. Car voici seulement cinq, sept ou huit ans, les producteurs de ces formules choc particulièrement lugubres dont sont si friands les médias se complaisaient encore à en émailler le catalogue thématique du processus d'unification. Par exemple : " liquidation totale de la RDA », " discrimination des Allemands de l'est en matière de salaires ", "ségrégationn » systématique des nouveaux Länder et
Uwe Kammann, journaliste, a été rédacteur en chef de epd medien (1984-2005), membre du jury du Prix Grimme, avant d'être nommé directeur de l'Institut Grimme (Marl) qui décerne les prix Grimme-Preis et Grimme Online Award récompensant la qualité des émissions de TV et des articles de la presse en ligne.
RDA :

la « bonne » Allemagne? 
Un préjugé

20 ans après un bilan globalement positif

Une fierté non dénuée d'humour

Une identité aujourd'hui sereine des Allemands de l'est, "destruction au bulldozer » de prétendus acquis sociaux ou encore "dévastation » culturelle et mentale... Présentée ainsi avec force jugements à l'emporte-pièce, l'ex-RDA ne pouvait alors apparaître au fond que comme la meilleure des deux Allemagne, comme un petit Chaperon rouge promis à un bel avenir, mais hélas dévoré par le grand méchant Loup capitaliste.

Pourtant, quiconque voulait bien regarder les réalités en face, sans laisser travestir son regard par l'idéologie (mais aussi quiconque cherchait à cacher sous une belle rhétorique son propre aveuglement, ses erreurs d'appréciation ou ses erreurs d'interprétation politique) savait depuis toujours que cette image de la « mauvaise » République fédérale capitaliste se jetant sur la «bonne » petite Allemagne pour la dévorer goulûment n'a jamais été et ne sera jamais qu'une vision déformée de la réalité. Pour le dire d'un mot : cette image est tout simplement fausse. Mais cette conscience s'accompagnait (s'accompagne) aussi de ce constat: sa dramaturgie simpliste ne présentant les choses qu'en noir ou blanc est évidemment idéale pour étayer les préjugés - un réflexe courant lorsqu'il s'agit d'une thématique aussi chargée d'émotions et portant sa part de rêve que l'est la réunification.

Mais le plus étonnant en cette année 20 du processus d'unification est ceci : c'est presque en chœur que les médias en tirent un bilan globalement positif. Certes, ils évoquent toujours le «caractère inachevé » de l'Unité, mais non plus sous la forme d'un murmure à tonalité négative ; ils le font plutôt en rappelant que l'Unité est une haute mission, et une mission qui exige un effort permanent car il faudra bien trois générations pour que les anciennes différences entre les sociétés, les politiques, les individus, puissent s'abraser comme elles se sont lissées au sein de cet entrelacs de régions qu'est la République fédérale. Dans Das Parlament, un hebdomadaire proche du pouvoir (il est édité par le Bundestag) et néanmoins hautement réputé, l'ancien écrivain de la RDA, Erich Loest (une autorité respectée à l'est comme à l'ouest) parvenait à cette conclusion au début de septembre : “En 1990, la différence entre l'Allemagne de l'Est et de l'Ouest était immense » alors qu'aujourd'hui, 20 ans après, les différences inter-allemandes "se sont déplacées ", s'intégrant dans le tableau de la normalité d'une Allemagne plurielle faite d'identités et particularités régionales.

Dans un éditorial ouvrant un bilan approfondi de l'Unité allemande paru dans la presse, se trouve cité en qualité de témoin principal du succès accompli l'ancien ministre fédéral des Affaires étrangères, Hans-Dietrich Genscher. Avec pour argument central le constat que l'unité intérieure (dans les têtes) est bien plus proche de l'achèvement qu'on ne le croit souvent. Les jeunes sont conscients de leur avenir commun. Quant à l'hebdomadaire libéral Die Zeit (dont un des directeurs de la publication n'est autre que l'ex-chancelier SPD Helmut Schmidt), il préféra publier sa reconnaissance d'un processus largement plus couronné de succès qu'il ne pensait en la présentant sous une forme ironique plus vendeuse, plaçant cette manchette en une de son cahier Culture : " 20 points que nous réussirons mieux lors de la prochaine réunification ».

Il exprimait ce faisant la tonalité générale des récits, analyses, descriptions et évaluations publiés dans la presse écrite comme dans les médias électroniques: une certaine sérénité, voire une auto-admiration non déguisée. Et qui reposait sur des réfexions qu'on pourrait résumer ainsi : au fond, nous avons fait là du bon travail ; enfin, il nous est permis à nouveau, à nous autres Allemands, de nous sentir bien dans notre peau et : oui, pour la première fois depuis 150 ans naît une identité allemande, faite de naturel et de sérénité, pour la première fois, la plupart des Allemands ont réussi à se trouver. Avec une conscience naturelle, fruit de l'évidence, une profonde détente intérieure après cet enchaînement de crispations et divisions qui ont marqué l'histoire et mené la société allemande jusqu'au bord de l'auto-destruction totale.

Une telle approche positive - amplifiée à la surprise générale par tous ces moments de joie festive lors des Coupes du Monde de football 2006 et 2010 - était jusque-là inconnue dans cette ampleur comme ses multiples manifestations. Et 
elle s'est transmise - sous l'effet de gigantesque catalyseur et d'amplificateur des émotions qu'a eu ce "patriotisme-fiesta » si décontracté qui s'était fait jour au moment de la Coupe du Monde à domicile - sensiblement même aux médias, alors que ces manifestations identitaires les avaient d'abord laissés pour le moins sceptiques. Ce faisant, les médias ont eux-mêmes développé une nouvelle approche dans l'accompagnement du processus d'unification et jusque dans l'appréciation même de l'Unité allemande : eux aussi se montrent désormais moins crispés, plus bienveillants, plus sereins. Et c'est ainsi qu'on peut observer comme une synchronisation croissante de ce que pense et ressent l'opinion : 20 ans après la chute du Mur, on perçoit une tonalité entièrement nouvelle, une nouvelle posture - les dents ne grincent plus. Comme si s'était produit dans les médias quelque chose comme une auto-vérification - et ce, dans une société où, surtout dans les années d'après-guerre, les médias ont joué un rôle décisif...

\section{Les médias : " medium et facteur 》 de l'opinion}

II y a des vérités aussi incontestées qu'élémentaires, dont celle-ci : le monde moderne ne fonctionnerait pas sans les médias. Et encore moins une démocratie moderne dont l'idéaltype veut qu'elle soit ouverte. Les médias sont tout simplement les courroies de transmission de toute société. Ils le sont quelle que soit leur structure et même quand ils ne sont pas au-dessus de tout soupçon. C'est justement ce rôle, pour le dire d'entrée, qu'on joué les médias en Allemagne dans ce moment où se conjugaient évolutions internationales et mouvements nationaux, amenant le monde à un tournant fondamental pour le plonger dans une nouvelle ère - de manière visible et sensible pour tous. Et dans tous les moments de cette époque à la fois agitée et passionnante, les médias - au sens le plus large du terme : des médias de masse que sont la télévision, la radio et la presse, jusqu'au théâtre, en passant par le livre et le cinéma - étaient directement impliqués, prenant une part active aux évolutions.

Ils assumèrent là exactement cette double caractéristique foncière que le Tribunal constitutionnel fédéral avait jadis attribué sous une formule quelque peu tautologique aux médias électroniques en définissant leur rôle comme " medium et facteur » de l'opinion publique. Traduit en termes plus simples, cela veut dire qu'en tant que vecteurs, les médias enregistrent quelque chose, restituent cette chose comme un miroir, créant un cadre qui en concentre la perception et la transmission. Et de l'autre côté, c'est justement grâce à cette diffusion - qui implique qu'ils hiérarchisent et commentent, désignent et focalisent ce qu'ils transmettent - que les médias font évoluer le monde.

On peut illustrer cette fonction par l'image d'un parallélogramme de forces où chaque ligne ou vecteur, en l'occurrence chaque voix qui s'élève dans un but précis, contribue à faire, par sa force particulière et la direction visée, que ces millions de vecteurs ou de flèches médiatiques et flèches d'opinion se rejoignent pour se condenser en une ligne ou flèche directrice qui agit sur le cadre et détermine ainsi la forme de cet ensemble. Appliqué à une société, cela signifie que la manière dont elle se présente au sein du parallélogramme qui lui est propre dépend de toutes ces innombrables énergies individuelles qui agissent sur elle.

Ce même principe permet aussi de mieux comprendre pourquoi, dans la Constitution allemande, figurent en position privilégiée, c'est-à-dire au $5^{\mathrm{e}}$ rang des droits fondamentaux, ces quelques phrases qui expriment l'essence de notre société démocratique et lui confèrent vie : «Chacun a le droit d'exprimer et de diffuser librement son opinion par la parole, par l'écrit et par l'image, et de s'informer sans entraves aux sources qui sont accessibles à tous. La liberté de la presse et la liberté d'informer par la radio, la télévision et le cinéma sont garanties. II n'y a pas de censure » (art. 5 de la Loi fondamentale).

C'est sur ces phrases si limpides qui font partie des fondements sacrés, inviolables, de la République fédérale, que repose la success story de la RFA (Petersen, 2009) dont le $60^{\circ}$ anniversaire fut célébré dans un si frappant élan de com-
Double fonction, constitutionelle, des médias

La démocratie allemande repose sur leur fonction de critique ... 
... et sur leur pluralisme

Les médias ont joué un rôle majeur dans le processus d'unification

munion l'an dernier. Après les années de barbarie nazie où le pouvoir ne concédait aux médias qu'un unique rôle - celui d'instrument de propagande inféodé au régime -, ces phrases tiraient les enseignements pratiques de cette expérience : le rôle des médias doit être d'accompagner la vie politique, sociale et culturelle en la relatant, l'analysant et la commentant. Et ce en tant qu'instance fondée sur la distance avec le pouvoir pour qu'elle puisse assumer sa fonction de critique et permettre à la société de dialoguer avec elle-même.

Aujourd'hui, plus de 60 ans après, le constat s'impose : cet objectif a été largement atteint, même si ce processus n'a bien sûr pas été ni n'est encore exempt de nombre d'égarements ou d'erreurs, d'âpres affrontements idéologiques ou de féroces batailles entre concurrents sur le marché. II n'en reste pas moins que chacun des grands secteurs - de la presse, en passant par l'audiovisuel et le cinéma, et jusqu'au livre (je n'inclus pas ici les nouvelles formes de médias) - a pu développer ses propres points forts et sa contribution à un paysage médiatique qui a su conquérir, grâce au pluralisme comme à la qualité que lui attestent de nombreux observateurs, une place dans le palmarès de tête des comparaisons internationales.

Cette qualité globale qui s'était progressivement développée dans la RFA d'avant l'Unité et qui est la résultante de l'ensemble de ses composantes, a joué un rôle majeur pour permettre à la société allemande de trouver son identité grâce à un perpétuel processus d'apprentissage : d'abord dans la prise de conscience, souvent impulsée de l'extérieur, de la barbarie et de la terreur du passé immédiat, puis dans la revendication violente d'une remise en question du statu quo de la société au cours des années 1960/70, et enfin dans une nouvelle sérénité identitaire (Petersen, 2009). Ce processus s'est effectué au sein d'un système démocratique foncièrement stable où les médias remplissent correctement dans l'ensemble le rôle de charnière que leur confère la Constitution.

Or - et c'est là la première thèse centrale de la présente contribution - cette même configuration positive a rempli son rôle quand eurent à se réunir, voici deux décennies, deux systèmes qu'on ne pouvait imaginer plus différents. Ils avaient cependant une grande communauté culturelle : le lien de la langue. Sans parler d'un passé commun. Bien sûr, on peut toujours mettre de l'eau dans son vin quand on évoque globalement les mérites d'un système. II n'en reste pas moins que nombre d'observateurs patentés des médias, rompus à l'exercice des comparaisons internationales comme Isabelle Bourgeois en France, attestent de la fonctionalité de l'interaction entre médias et sociétés en Allemagne, avant ou après l'Unité. Elle constate ainsi que la République fédérale est " une démocratie construite sur la libre circulation de l'information » et souligne que l'Allemagne est une " société civile organisée » avec de multiples capitales et centres de pouvoir et de décision (Bourgeois, 2010). Parmi les principaux facteurs expliquant le bon fonctionnement de l'interaction entre médias et société, elle cite l'organisation fédérale du pays, le système des établissements de radiodiffusion de droit public (régionaux et autonomes), de même que la puissance des éditeurs de presse dont le capital n'est pas détenu par des groupes étrangers aux médias (comme c'est le cas par exemple en France). Plus fondamentalement encore, c'est « l'indépendance structurelle » du pouvoir médiatique par rapport au pouvoir politique (Bourgeois, 2009/10) qui, parce qu'elle s'applique largement au quotidien, explique le bon fonctionnement de cette interaction entre médias et société.

\section{Pluralisme structurel des pouvoirs et des médias}

RFA et RDA : deux conceptions du rôle des médias
Le principe pivot sur lequel repose ce paysage médiatique - et que le Tribunal constitutionel fédéral a rappelé à de nombreuses reprises, l'érigeant en norme pour un audiovisuel qui fut très longtemps exclusivement public (c'est-à-dire, dans l'acception allemande, appartenant à la collectivité des citoyens, donc de la société) - est le pluralisme. Cette norme de droit fondatrice, essentielle pour la reconstruction de la République fédérale, était aussi le noble fruit de l'expérience, 
tirant un trait sur un passé de dictature où le pilotage central avait abouti à une large mise au pas, où interprétation, information, orientation et directives étaient synchrones et homogènes. Un tel pilotage central, décidé, mis en œuvre et contrôlé par l'Etat, caractérisait aussi le système médiatique de la RDA, à quelques réserves ou particularités spécifiques près, bien sûr.

Dans ses grandes lignes, une distinction fondamentale démarquait jusqu'à l'automne 1989 le système médiatique d'une Allemagne de l'Ouest démocratique et celui d'une Allemagne de l'Est régie par la préfiguration d'un socialisme d'Etat. Dans l'acception de l'Ouest, les médias étaient (et sont toujours) considérés, sous leur vision idéaltypique, comme des moyens permettant aux citoyens de s'éclairer eux-mêmes, en pleine responsabilité ; et il appartient à une société ainsi formée par les médias et constituée grâce à eux de contrôler le politique. Dans l'acception de l'Est, les médias étaient considérés, toujours sous la forme d'idéaltype, comme un instrument de pilotage de la société par l'Etat et ses institutions.

Toutefois, comparé aux systèmes de pilotage du même type, l'Allemagne orientale se dinstinguait par une sorte de correctif qui le relativisait : à savoir un système concurrent (celui de l'Allemagne occidentale limitrophe) dans la même langue, reposant sur la libre circulation de l'information, et qui de ce fait livrait quasi à domicile un pluralisme de valeurs, alors que l'Etat socialiste aurait tant souhaité rendre sa 'maison' imperméable aux influences extérieures. Mais comme, par principe, les informations transmises par les ondes traversent portes et fenêtres même quand elles sont fermées, permettant la comparaison et un jugement différencié, ce flux interdit à la situation de se figer.

Aujour'dhui, en 2010, sur le réseau mondial qu'est la Toile, nous connaissons à nouveau une situation comparable, alors que se livrent une concurrence avivée des types de médias très différents, véhiculant des approches multiples, autant de miroirs qui ne cessent de refléter différemment la réalité, et autant de nouveaux forums où se forment en permanence des visions nouvelles et multiples visions du monde. Ces évolutions ont elles aussi modifié les règles du jeu médiatique, dans le sens d'une perpétuelle révolution interne entretenant la relativisation de chacune des approches ainsi en compétition, et peut-être même au sens d'une inflation immanente des vérités et valeurs des médias. Or les exigences ainsi accrues entretiennent et entraînent bien sûr, elles aussi, la capacité de chacun à adapter sa pensée, à changer d'approche, à apprendre les règles du jeu indispensables à la survie et qui consistent à comparer les informations, leurs sources et les lectures proposées afin de se forger sa propre opinion et d'être ainsi capable d'agir ou, au moins, d'accroître sa capacité à agir.

L'organisation fédérale prononcée de la RFA a eu une contribution majeure dans le développement du pluralisme, puisqu'elle instaure un équilibre quasi structurel des centres de pouvoir et d'influence. Cela a toujours été le cas de la presse où les principaux journaux - comme les quotidiens Frankfurter Allgemeine Zeitung, Süddeutsche Zeitung, Tagesspiegel, Die Welt ou Stuttgarter Zeitung - paraissent en des lieux géographiques différents: Francfort, Munich, Berlin ou Stuttgart. Quant à Hambourg, c'est le lieu de production des grands hebdomadaires que sont Der Spiegel, Die Zeit et stern. Mais la radio et la télévision aussi sont l'émanation de cette structure polycentrique du pays, qu'il s'agisse de leur genèse historique, de la structure des établissements ou de leur culture; Berlin (donc le siège du gouvernement fédéral) n'est qu'un lieu parmi d'autres dans ce réseau. Les Länder exercent un fort pouvoir sur l'audiovisuel de droit public puisqu'ils disposent chacun sur son territoire des compétences législatives en ce qui concerne les grandes lignes de l'organisation des opérateurs qui sont par ailleurs et par principe indépendants de l'Etat dans leur gestion comme leurs programmes. Quant aux deux plus grands groupes d'opérateurs privés (RTL et Pro7Sat.1), ils ont leur fief respectivement à Cologne et Munich. C'est ainsi que se présente l'équilibre régional des pouvoirs médiatiques en Allemagne.

Et c'est justement à cet équilibre régional des pouvoirs médiatiques, ancré dans le fédéralisme, que revient le rôle de facteur clé dans la réunification. II explique
Ouest : les citoyens contrôlent le politique ; Est : l'Etat pilote la société via les médias

RDA : un pluralisme de facto importé par les ondes

RFA : un pluralisme institutionnel aussi

Il a joué un rôle clé dans l'unification 
pourquoi, malgré tous les antagonismes, malgré tous les écueils, malgré toutes les douloureuses récusations collectives et indiviuelles, malgré toutes les injustices commises dans le détail, la réunification a pu suivre son chemin. Et pourtant, ce chemin, il fallait le concevoir, le décider, le tracer et le paver; et faire le plus souvent tout cela en même temps.

Certains, mus par la ferme volonté de l'action, auraient assurément préféré monopoliser ces médias qui accompagnaient le processus d'unification de leurs critiques et polémiques, pour en faire un 'commando de l'unification'. Certains leur auraient volontiers dicté à partir de quand il aurait fallu cesser d'évoquer les "Ossis » et les «Wessis », la «Stasi », les " chevaliers d'industrie », "les conquistadors des bananes » ou le "Mur dans les têtes », quand il eût été de leur devoir de louer la Treuhand en tant qu'institution dotée du pouvoir d'exécuter le processus de transformation économique, et quand ils auraient dû faire l'éloge des «Krauses » et «Seiters » - nommés ici en tant que prototypes de ministres de l'est et de l'ouest en charge du processus d'unification - au lieu d'accompagner leur action avec un méticuleux sens critique.

\section{Le débat sur le processus d'unification était une res publica}

Or c'est justement parce que la relation et/ou transmission du processus d'unification dans l'espace public fut si transparente, qu'elle fut sans concession et qu'elle ne recula pas devant les conflits, et ce tous azimuts, que les acteurs ont agi de leur mieux. Car sans nul doute: pour déployer toute son énergie dans l'action, chacun a besoin d'air frais, et en permanence ; et donc du débat, de la confrontation des schémas de pensée.

Aujourd'hui, 20 ans après, on a du mal à imaginer combien les débats ont parfois été virulents, et pas seulement entre des camps sommairement partagés entre est et ouest, mais bien plutôt au sein même des innombrables groupes, groupuscules, formations, mouvements politiques, partout à l'est même, à l'ouest même, par-delà les points cardinaux et l'ancienne ligne de démarcation.

Cible de la haine des médias : R. Mühlfenzl, chargé de liquider l'office audiovisuel de la RDA
Une personnalité illustre à la perfection la complexité de l'affrontement de ces intérêts : Rudolf Mühlfenzl, ancien responsable (organisation et programmes) tant dans l'audiovisuel public que privé. Il avait été chargé (par le chancelier Helmut Kohl personnellement) du poste de plénipotentiaire du gouvernement fédéral pour l'audiovisuel avec pour mission de liquider un organisme appelé « l'Institution » (die Einrichtung). Cette appellation quelconque et banale désignait l'audiovisuel de l'ex-RDA, c'est-à-dire toutes les chaînes et radios anciennement contrôlées par l'Etat, pilotées depuis Berlin, et sises à Berlin, capitale de la RDA. Autrement dit, il s'agissait d'un appareil d'Etat occupant plus de 14000 salariés, du directeur général (Generalintendant) jusqu'aux jardinières d'enfants et cuisinières, en passant par les directeurs de programmes et les journalistes. Ce qui était particulier au transfert de cette administration médiatique, dont la gestion était centralisée et qui était placée sous le contrôle du Politbureau, dans cette "Einrichtung » instituée ad hoc, c'était qu'elle concernait en réalité des êtres humains, chacun avec sa biographie, ses angoisses et ses espoirs, et dont le destin professionnel et humain dépendait maintenant d'un plénipotentiaire nommé Mühlfenzl.

C'est ainsi que ce Rudolf Mühlfenzl s'attira la haine pas seulement des salariés de l'audiovisuel de l'ex-RDA ou de nombre de ces intellectuels est-allemands qui prônaient un régime nouveau, à mi-chemin entre capitalisme et socialisme - la " troisième voie ». Les médias de l'ouest aussi, qui accompagnaient ce processus de liquidation de l'audiovisuel de l'ex-RDA, achevé à la fin 1991, critiquèrent vivement $R$. Mühlfenzl et lui manifestèrent leur hostilité. Pour résumer, quitte à forcer le trait : il était présenté généralement comme un exécuteur de sang froid pourchassant impitoyablement toute tentative de réfléchir à ce qu'aurait pu être une «troisième voie », et donc comme le fossoyeur médiatique d'un nouveau système audiovisuel qui aurait été bâti sur un modèle propre et aurait intégré l'expérience de l'est. 
On le présentait donc comme le fossoyeur de tous ces objectifs, de toutes ces visions d'une organisation possible, qui animaient à l'est les défenseurs des droits de l'Homme qui prônaient une RDA réformée, c'est-à-dire un pays autonome, à moderniser et à démocratiser pas à pas, et qui n'aurait pas été soumis aux normes ni aux modes d'organisation de la République fédérale (ils étaient rassemblés notamment au sein de l'instance de concertation Runder Tisch: «Table ronde ", constituée en décembre 1989). Aujourd'hui, avec le recul, on ne peut que 'réhabiliter' l'image de $\mathrm{R}$. Mühlfenzl : il a enseveli, et à juste titre, la structure audiovisuelle du complexe politico-propagandiste des fonctionnaires du Parti. Dans une récente interview accordée à epd medien, Roland Tichy, qui était alors son bras droit, décrit le déroulement des opérations, ainsi que les mécanismes comme les secrets alors apparus au grand jour. Sa conclusion : il n'y avait pas d'autre solution qu'une rupture totale, tant cet appareil était imprégné par les usages des fonctionnaires du Parti.

Qu'il soit ici permis une remarque personnelle à l'auteur, dont les révolutions de cette époque, les événements qui se succédaient à un rythme effréné, les débats fondamentaux et profondes mutations en cours ont marqué les années les plus passionnantes et les plus productives de sa propre vie professionnelle - médiatique. J'étais à l'époque rédacteur en chef de la revue spécialisée sur les médias epd medien (elle s'appelait alors Kirche und Rundfunk). Cette revue bihebdomadaire suivait jusque-là essentiellement l'évolution du paysage audiovisuel de la République fédérale sous ses divers aspects : politique des médias, économie des médias, structure du paysage et critique des programmes. Mais à cette époque, alors que du jour au lendemain tout semblait changer, les constellations d'acteurs, et jusqu'à leurs fondements mêmes, ce qui valait avant tout pour les médias de la RDA valait aussi pour notre petite équipe rédactionnelle : nous travaillions dans une atmosphère fiévreuse et passionnée. Jamais auparavant, notre rédaction n'avait ressenti aussi intensément (toutes les autres pareillement, à l'ouest comme à l'est) que le travail journalistique permettait de changer les choses ; nous le pouvions, nous le voulions et nous le devions.

C'était une chance unique à nos yeux que de pouvoir, dans cette situation historique, contribuer à forger l'avenir, c'est-à-dire à le faire en livrant une information objective et digne de foi, en relatant les événements dans le plus haut respect de la véracité des faits, pour permettre l'articulation et la diffusion des principales positions exprimées par les principales parties impliquées. Nous étions mus par l'idée, et même l'espoir, que s'offrait là à nous l'opportunité d'un renouveau et qu'il était maintenant possible de faire évoluer dans un sens positif une République fédérale largement ankylosée. Si je me souviens bien, la critique à l'encontre d'une 'vieille' République fédérale perçue ou publiquement présentée comme réfractaire aux réformes, en proie à l'immobilisme, était alors dans l'air du temps au sein de toutes rédactions, commune à toutes les approches, qu'elles soient conservatrices, libérales ou sociales.

Ces bouleversements qui avaient lieu dans un laps de temps très bref et qui remettaient en question tout ce qui semblait jusque-là figé pour l'éternité pouvaient aboutir à des situations cocasses. C'est ainsi que, au début de 1990, tout juste trois mois après la chute du Mur, un journaliste (en l'occurrence l'auteur) se trouva soudain dans le bureau du Medienkontrollrat face à un homme portant une longue barbe... (c'était le signe distinctif des intellectuels de la RDA). Ce barbu donc, Andreas Graf, était en réalité chercheur (spécialiste de l'anarchisme !) et avait été hissé via la «Table ronde » à son poste de responsabilité au sein du Medienkontrollrat (mot à mot: «Conseil de contrôle des médias »; sic !), une institution créée par la Volkskammer dans l'improvisation la plus totale et chargée de veiller au renouveau démocratique des médias dans ce qui était encore la RDA (il siégea pour la première fois le 13 février).

Tout dans ce renouveau des médias était mémorable. Car ce Medienkontrollrat était hébergé... dans les locaux du ministère de l'Information de la RDA. Or avant la dictature de la RDA, le même immeuble au centre de Berlin (Est) abritait... le
On lui reprochait d'être le fossoyeur de la " troisième voie "

Pour les médias, l'ouverture de la RDA est une chance unique de participer activement au processus

RDA : un renouveau menant parfois à des situations cocasses 
bureau du ministre nazi et propagandiste en chef du régime hitlérien : Joseph Goebbels. Un fait qui avait de quoi plonger dans une perplexité extrême même les journalistes occidentaux les plus chevronnés. Et quel ne fut pas leur étonnement lorsqu'ils durent constater que, dans ces lieux stratégiques du pouvoir de la RDA, les téléphones étaient aussi rares que les télécopieurs, et que l'information mettait un temps interminable à circuler dans les rouages tortueux d'un système de communication d'un autre âge.

Friedrich Wilhelm von Sell, qui avait dirigé l'établissement de radiodiffusion Westdeutscher Rundfunk (Cologne; le plus grand d'Allemagne) et qui se trouvait soudain, au début des années 1990, investi d'une sorte de mission d'aide au développement occidental à la tête du nouvel établissement Ostdeutscher Rundfunk, minuscule en comparaison (et logé dans les 'baraquements de Babelsberg' qui étaient une sorte de P.C. pour la reconstruction de l'audiovisuel est-allemand), dut demander à son assistant de mettre en marche un poste de TSF militaire pour joindre ses têtes de pont à Cologne et Düsseldorf... C'était le règne de l'improvisation. Mais en même temps, il vous donnait le sentiment d'avoir des ailes, on se sentait engagé à l'extrême dans ce nouveau départ vers l'inconnu qu'on saluait comme une chance malgré toutes les incertitudes.

\section{Le pouvoir des images}

La TV ouest-allemande a eu un rôle amplificateur et protecteur

Face aux caméras, les Allemands de l'Est se mettent en scène

La chute du Mur : un méga-event !
Dans cette révolution pacifique de la RDA, les médias ont indéniablement joué un rôle d'entraînement. Par exemple, parce que, en octobre 1989, les habitants de Leipzig pouvaient suivre à la TV ouest-allemande leurs «manifestations du lun$d i$ », voir leur mouvement s'amplifier sur le grand boulevard Ringstraße et constater qu'on ne tirait pas sur la foule. Ce sont ces images, diffusées par les journaux télévisés de l'ouest, qui en firent un mouvement de masse, ce qui anima en retour les réflexions des télépectateurs de l'est comme de l'ouest, les rendant méditatifs et certainement pleins d'espoir aussi, et qui limitèrent fortement la latitude d'action des acteurs politiques à l'Est. Car ceux-ci savaient que toute manifestation de violence ferait immédiatement le tour du monde sous forme d'images télévisées. Cet espace public potentiellement global les plaçait devant des choix cornéliens, alors qu'à l'inverse, il plaçait largement sous sa protection les citoyens.

Les citoyens et téléspectateurs est-allemands se mirent dès lors à produire euxmêmes des images reflétant les mutations de la société et les impulsions politiques. Ces quantités impressionnantes de drapeaux noir-rouge-or que la foule agitait sciemment devant l'opéra de Leipzig (au fait, d'où provenaient-ils, ces drapeaux sans le marteau, le compas et les épis, symboles officiels de la RDA ?), elles disaient aux téléspectateurs à l'ouest comme à l'est de l'Allemagne : nos revendications vont plus loin encore, nous voulons une Allemagne unie. Or je me rappelle que cela n'était pas forcément pour plaire à un certain nombre de journalistes TV de l'ouest bien ancrés dans les cercles traditionnels de la capitale (Bonn). Ni non plus à un nombre non négligeable de mes confrères de la presse écrite. Ce qui les amena souvent, dans leurs informations et commentaires, à minorer l'ampleur des événements - et ce, contrairement à l'évidence même. La perspective d'une éventuelle réunification ? Plutôt une vision d'horreur dans l'esprit de nombreux journalistes ouest-allemands...

Et quand finalement le Mur tomba, sa chute elle aussi était plus qu'idéalement médiatique : c'était un méga-event! Qu'il était télégénique, ce Mur avec ses tags multicolores côté Ouest, décor prenant vie grâce au mouvement de la foule. Action! Des mains qui tapotent les Trabant, les larmes et le champagne qui mousse, les embrassades, les cris : " c'est inimaginable ! ”, les morceaux de Mur qu'on casse, les coups de marteau sur le symbole de la partition et aussi de la mort... C'était plus beau et plus enivrant que la plus belle des mises en scène. Une fête qui s'auto-entretient, diffusée en boucle sur toutes les chaînes, et une boucle qui, de surcroît, s'enrichit de minute en minute. A une seule reprise seulement, plus tard, on revivra avec la même intensité le choc des images : quand s'effondrent les Twin Towers à New York. 
Que la télévision a contribué à la chute du Mur et l'a même accélérée s'impose à l'évidence (voir Bourgeois, 2009 et de Peretti, 2009). Le coup de grâce lui a été porté par Hans Joachim Friedrichs, journaliste respecté, blanchi sous le harnais et présentateur-vedette du magazine d'informations télévisées Tagesthemen de la Une (Ouest). Le soir du 9 novembre, il avait forcé le trait en rapportant les célèbres phrases marmonnées par Günther Schabowski, membre du Politbureau, à propos de la nouvelle réglementation des voyages : " La RDA a annoncé que ses frontières sont immédiatement ouvertes à tout un chacun. Les portes dans le Mur sont largement ouvertes ». La simple allégation, par un journaliste, d'une ouverture que les dirigeants de la RDA n'avaient pas l'intention de prévoir dans cette ampleur, a suffi par se transmuer presque immédiatement en réalité.

A la fête de la chute du Mur a succédé la 'Grande investigation'. Elle fut souvent empreinte de stupéfaction et reste déconcertante. Alors qu'avant la chute du Mur et avant la politique réformatrice menée par le président de l'Union soviétique, Mikhaïl Gorbatchov, ils avaient traité la RDA non sans quelque bienveillance (l'auteur force là quelque peu le trait dans un souci de clarté, tout en étant conscient que sa présentation peut prêter le flanc à la critique), les journalistes ouestallemands ont brutalement rompu avec ce ménagement. Face aux caméras des essaims de correspondants et des équipes TV envoyées pour couvrir la RDA, celle-ci n'était plus qu'une nation catastrophée, un pays depuis longtemps en déclin. On trouva et on diffusa maintenant des images montrant des lacs contaminés par des produits chimiques ou des conditions de production aux effets dévastateurs sur l'environnement telles qu'elles caractérisaient l'assemblage des Trabant, véhicules d'une obsolescence notoire et à la carosserie toute de plastique. Or cette vision, véhiculée à l'unission, d'un Etat en pleine décomposition, visible de l'extérieur à ces signes de délabrement, était souvent teintée d'opportunisme.

Beaucoup d'éléments que contenaient maintenant les reportages (TV, radio, presse écrite) n'étaient que folles exagérations portées autant par la stupéfaction et l'incrédulité que par une indomptable volonté de changement. Elf 99, l'émission pour les jeunes lancée naguère à la télévision de la RDA et qui était idéologiquement conforme, se mit soudain à adopter un ton irrévérencieux, prit de l'audace et se lança dans l'investigation. Les images qu'elle rapporta de Wandlitz - « l'enclave des bonzes ", comme on surnommait cette résidence -, contribuèrent largement à saper le peu d'autorité restant aux anciens fonctionnaires du parti qui y menaient une existence privilégiée à l'abri du regard de la population. Des machines à laver de la marque Miele pour prouver leur décadence et leur hypocrysie - des arguments visuels irréfutables, du moins à l'Est. Ce sont toutes ces accréditations de ce qui était foncièrement inconcevable (qu'était-il réellement possible de savoir en RDA ?) qui accélérèrent en la renforçant la pression sur le régime. Celui-ci ne put y résister.

\section{Audiovisuel : le fédéralisme s'impose}

Qu'advint-il du système audiovisuel ? On vit naturellement s'engager immédiatement une féroce bataille : à quoi ressemblerait-il, comment et sous quelle forme intégrerait-il dans ses structures les nouvelles libertés de l'information que conquéraient (et goûtaient) de jour en jour les journalistes et responsables de programmes, tout en garantissant leur pérennité ? Tout aussi évidente était la grande méfiance que ces réflexions suscitaient surtout dans la nouvelle classe politique qui était en train de s'organiser au sein de mouvements très disparates. Mais la plus lourde hypothèque pour les salariés est-allemands s'imposait d'ellemême : elle résidait dans le centralisme de la radiodiffusion de la RDA, son histoire, ses liens étroits avec les organes de l'Etat.

Et même une personnalité d'envergure comme l'était Hans Bentzien, le dernier directeur général du Deutscher Fernsehfunk comme avait été pompeusement rebaptisée entre temps l'ancienne TV de la RDA, dut reconnaître son impuissance face aux habitudes prises de ce fait. C'est avec une admirable énergie que $H$. Bentzien, au cours de cette phase de transition et d'ouverture parfois anarchique,
Après la fête, la 'Grande investigation'
H. Bentzien lutte pour établir un système public à l'est 
Mais l'audiovisuel de la RDA intègre le système de l'ouest

Le fédéralisme audiovisue permet aux cultures régionales de s'exprimer et de s'épanouir

Mais le Mitteldeutscher Rundfunk s'attire les moqueries lutta pour une «troisième voie » propre à l'audiovisuel sur le territoire de ce qui était encore la RDA, pour une troisième structure de droit public aux côtés de l'ARD et de la ZDF à l'Ouest. Pourtant, tout dans sa carrière - il avait été ministre des cultes et avait dirigé les éditions Junge Welt - le prédestinait à véhiculer et à mettre en pratique le mode de vie et les valeurs de la RDA. Mais très vite, bien avant que ne prenne de l'ampleur la revendication d'une Allemagne unie, officiellement exaucée quelques mois plus tard, il dut se rendre à l'évidence que ceux-ci appartenaient déjà au passé. Une autre approche politique avait pris le relais, s'exprimant dans la volonté de miser à nouveau sur des structures régionales, sur le fédéralisme - comme si, même sur le territoire d'une RDA (re)centralisée, les Länder n'avaient jamais cessé d'exister dans leur ancienne configuration.

Et c'est ainsi que finalement naquit, reflet structurel de l'acte d'adhésion de la RDA à la République fédérale, un système audiovisuel qui n'était lui-même que le reflet de l'évolution de la radiodiffusion dans l'Allemagne de l'ouest d'après-guerre dans un Etat fédéral : le réseau polycentrique des établissements fut simplement étendu, intégrant de nouveaux éléments et de nouvelles approches (voir dans ce numéro l'analyse d'l. Bourgeois). Nombreux furent ceux regrettant que, de la sorte se trouvait trop vite canalisé, voire réfréné, l'élan de renouveau des rédactions de la TV de l'ex-RDA. On jugeait cependant généralement défendable le fait que, dans le cadre de la vérification des biographies professionnelles qui s'était rapidement mise en place, soient limogés les anciens cadres investis d'une mission de propagande. Seules étaient mises en doute les méthodes employées dans ces vérifications, de même que leur faible taux de succès (un seul cadre démasqué pour plusieurs miliers de dossiers vérifiés).

A posteriori toutefois, il faut bien reconnaitre que, dans l'ensemble, le processus de transformation a bien mieux fonctionné qu'on ne croyait. Et que les particularités géographiques comme les cultures des nouveaux Länder de l'est aient pu trouver leur place et leur sens à la fois dans le paysage audiovisuel de l'Allemagne unie prouve une fois de plus l'ouverture comme la souplesse qui caractérisent cette Constitution de l'audiovisuel basée sur le fédéralisme qui s'est depuis longtemps établie à l'ouest et qui n'a cessé d'être affinée. Le Land de Mecklembourg-Poméranie décida même d'adhérer au Norddeutscher Rundkunk, établissement de droit public commun à trois Länder (Hambourg, Basse-Saxe et Schleswig-Holstein) et de s'y associer en tant que quatrième, serrant ainsi les liens est-ouest dans le nord de l'Allemagne. De leur côté, les trois nouveaux Länder du sud-est (Saxe, Saxe-Anhalt et Thuringe) joignirent leurs forces pour créer le Mitteldeutscher Rundfunk, tandis que le petit Land de Brandebourg, désireux de sortir de l'ombre de la puissance politique du Land de Berlin, chercha dans un premier temps à développer sa propre identité via son établissement: Ostdeutscher Rundfunk Brandenburg. Plus tard, les deux Länder fusionnèrent leurs établissements (donnant naissance au Rundfunk Berlin-Brandenburg), officialisant ainsi le lien médiatique logique (et raisonnable au plan économique) entre la capitale et sa périphérie. Au niveau des médias, l'union régionale a pu ainsi se faire, alors que le projet de l'unité politique entre Berlin et le Brandebourg - la fusion en un seul Land -, pourtant tout aussi judicieuse, a jusqu'ici échoué.

L'organisation de l'audiovisuel allemand selon les lois du fédéralisme a ainsi prouvé sa flexibilité et ce qui fait sa force. Concrètement, elle se traduit d'un côté par l'existence de structures régionales autonomes (Landesrundfunkanstalten), qu'elles soient l'établissement d'un seul Land ou de plusieurs, mais fédérées au sein d'un groupe de travail (dont le sigle est $A R D$ ) qui offre à chacune la possibilité d'y exercer des fonctions centrales de responsabilité ; d'autre part, par l'existence d'une structure commune à tous les Länder, l'établissement ZDF. Dans cette configuration, les identités régionales ne se trouvent ni diluées ni dissoutes ; au contraire, les particularismes - qu'il s'agisse de culture, de traditions ou de coutumes - peuvent déployer leurs propres forces et s'épanouir.

Mais cette culture des particularismes a fait aussi que par exemple le Mitteldeutscher Rundfunk, établissement commun à la Saxe, la Saxe-Anhalt et la Thuringe, 
s'est attiré un feu nourri de critiques et de railleries. Il a effectivement intégré dans ses structures et programmes nombre de concepts, de présentateurs et journalistes connus du temps de la RDA, plaçant sans réserve au centre de ses émissions les préoccupations et modes de vie spécifiques aux Allemands de l'est. Mais si ces Messieurs les critiques daignaient faire abstraction de leurs propres préférences en matière de programmes, peut-être finiraient-ils par reconnaître que c'était là, tout compte fait, la meilleure politique possible. Parce qu'elle intégrait et respectait justement tous ces éléments qui, dans une situation de bouleversement total, font que puisse se forger une nouvelle identité. Ce serait foncièrement méconnaître les réalités de la vie que de croire que dans de telles situations, la plupart des gens ne rêvent que de rupture et de renouveau. C'est plutôt l'inverse : face à l'irrémédiable, ils cherchent au contraire ce qui leur est familier, les rassure et leur permet de s'orienter.

Et quand on veut bien se rappeler tous ces congrès, séminaires, symposiums des premiers mois de 1990, tous ces groupes, lobbies ou hommes politiques de toutes obédiences qui cherchaient à esquisser les perspectives de développement de l'audiovisuel, on finit, avec le recul, par aboutir à cette conclusion : évidemment, il y avait de l'outrecuidance à l'ouest, maintes offensives de conquête de l'Est, et plus particulièrement des tentatives (comme celle du lobby des opérateurs privés de l'ouest) pour lancer un grand coup, s'accaparer les avantages matériels de la situation en mettant la main sur les réseaux de fréquences et accroître ainsi les parts de marché. Mais bien plus importante fut la portée de toutes les tentatives entreprises pour concevoir des modèles viables pour l'audiovisuel de l'Est, modèles visant à transférer à la RDA le concept d'un audiovisuel placé sous la responsabilité de la société. A l'époque, l'audiovisuel était encore le média de référence ...

Certaines de ces réflexions avaient une haute valeur symbolique. Ainsi, au printemps 1990, l'ARD avait choisi le Reichstag pour y débattre publiquement des modèles envisageables pour la structure future de l'audiovisuel. Les intervenants au podium étaient, du côté ouest-allemand, des personnalités à la haute réputation d'intégrité comme les anciens présidents d'établissements de l'ARD Hans Abich et Albert Scharf et, du côté est-allemand: Hans Bentzien, directeur général du Deutscher Fernsehfunk pour quelques jours encore, Manfred Becker, secrétaire d'Etat en charge des médias ; bien sûr , la science (ouest) aussi était présente, en la personne de Wolfgang Kleinwächter, chercheur en sciences de la communication. Ce symposium était d'une grande dignité et empreint d'un grand respect, tant par le choix du lieu ou des intervenants que par la qualité du dialogue. En tout cas, il n'y avait pas le moindre soupçon d'arrogance ni là, ni dans les symposiums organisés à Leipzig et Berlin par les syndicats des médias, ni dans le grand séminaire sur l'avenir de la structure audiovisuelle qui s'était tenu à l'Evangelische Akademie de Tutzing, ou le symposium Mainzer Tage der Fernsehkritik organisé par la ZDF. Tous s'étaient immédiatement consacrés à cette problématique et avaient invité à débattre les principaux représentants des médias de la RDA.

Pour en revenir au rôle de l'auteur de ces pages à la tête de la rédaction d'epd medien, publication de référence qui se devait de faire circuler l'information dans la branche : j'ai à l'époque accumulé les interviews des principaux acteurs et responsables à l'Est, dont Hans Bentzien, Manfred Becker, Gottfried Müller (ministre des médias), Gero Hammer (il ne fut directeur général que quelques jours avant d'être démasqué comme «collaborateur informel » de la Stasi), et bien sûr les hommes politiques et responsables des médias de l'Ouest. II était plus que justifié, dans cette situation d'urgence, dans ces conditions qui tenaient souvent de l'aventure, de mettre sur la table tout ce qu'il était possible de trouver en matière de réflexions pour alimenter ce qu'il faut bien appeler un gigantesque atelier théorique et pratique sur l'audiovisuel.

On a oublié l'incroyable vitesse à laquelle évoluaient alors les choses. Dieter Stolte, par exemple, qui présidait à cette époque la ZDF et qui n'avait nul pareil dans ses qualités de stratège visionnaire, avait décidé avec Hans Bentzien dès l'entrée en fonction de celui-ci que le Deutscher Fernsehfunk rejoindrait en qualité de
Un processus public de réflexion sur l'avenir de l'audiovisuel uni

Lieux hautement symboliques et débats d'une grande dignité

Un gigantesque atelier théorique et pratique

La TV de l'ex-RDA intègre 3sat 
Extension à l'est du réseau des autorités de régulation du secteur privé

Quand tout est bouleversé, les quotidiens permettent de s'orienter

II ne reste plus que 2 quotidiens supra-régionaux de l'ex-RDA

Un cliché mené à son paroxysme en 2000 : l'Allemagne de l'est, repaire de l'extrême-droite membre le consortium éditant la chaîne 3sat - coopération exemplaire des télévisions germanophones (ARD, ZDF, TV autrichienne et TV suisse alémanique) qui réalisent conjointement une chaîne culturelle hautement réputée.

L'évolution au cours des deux décennies a révélé que l'ouverture foncière d'un système audiovisuel organisé selon les règles du fédéralisme est une grande chance, puisqu'elle favorise la mise en réseau et une approche collective, de même qu'elle promeut l'autonomie à la fois de chacune de ses institutions et de leurs réalisations conjointes. Cela vaut pareillement pour l'autre pilier du système dual de la radiodiffusion : le secteur privé dont le cadre des activités est fixé dans ses grandes lignes par les autorités régionales de régulation (les Landesmedienanstanlten) qui contrôlent ce secteur au niveau de chaque Land. Ces centres de régulation, institués à l'est aussi, ont développé une coopération très productive ; on n'y distingue aucun déséquilibre systémique, aucune ligne de démarcation est-ouest, les Landesmedienanstalten des nouveaux Länder s'étant montrées dès le début à même de trouver leur place dans cette architecture. Et, tout comme à l'ouest, ces instances ont développé leurs caractéristiques et profils propres, toujours liés aussi à la présence de fortes personnalités à leur tête, mais sans jamais perdre des yeux l'objectif commun, à savoir veiller à créer des conditions favorables aux activités du secteur privé de l'audiovisuel.

\section{Presse : la normalité d'un marché concentré}

Pour résumer l'évolution de la presse écrite, le journaliste Frank Pergande (un ancien journaliste de RDA qui avait intégré la rédaction du Frankfurter Allgemeine Zeitung en 1998) avait voici peu eu dans ce quotidien cette formule pertinente : "monopoles, avant et après ». Autrement dit, à l'est, la presse a toujours pour base les anciens quotidiens de district du parti-Etat SED, mais rénovés. La Treuhandanstalt les avait mis en vente, et ils avaient rapidement trouvé acquéreur ; ils étaient bien ancrés dans leur région et représentaient pour les puissants groupes de presse de l'ouest une opportunité unique de croissance et de consolidation. Ces titres ont pu ainsi fidéliser leur lectorat puisqu'ils lui apportaient ces éléments d'orientation si nécessaires dans ces temps bouleversés.

Cela montre qu'à une époque où tout - de la législation au cadre de vie - change pratiquement du jour au lendemain, donc dans une époque en perpétuel état d'exception, le journal qu'on lit quotidiennement rassure par la certitude qu'il apporte, la confiance dans l'existence d'éléments invariables. Or cette fiabilité vaut nettement plus que le poids du passé, autrement dit le fait que le même journal avait été, hier encore, l'organe de la centrale du SED dans le district. Eux-mêmes pris dans un processus d'apprentissage et accumulant les expériences nouvelles, les quotidiens ont assurément rempli leur rôle : permettre au lecteur de s'orienter dans le monde. Et même aujourd'hui, confrontés à de profondes mutations recul accéléré d'une partie de leur lectorat, crise économique, remise en question de leur modèle économique, changement radical dans les habitudes et modes de consommation des médias - les quotidiens ne peuvent offrir que ce que tentent d'apporter partout dans le monde les quotidiens de facture classique.

Aujourd'hui, la concentration est indéniable : il ne reste plus à l'est que 17 rédactions autonomes, contre 100 à l'ouest (pour plus de 350 titres). II est dommage que, parmi les anciens quotidiens supra-régionaux de la RDA, seuls deux aient survécu : Neues Deutschland et Junge Welt. Et il est intéressant de constater que Super Illu, l'illustré quelque peu tapageur né au "temps des bananes ", à l'époque de la chute du Mur, se targue d'avoir aujourd'hui gagné en sériosité, bref, d'avoir mûri. Admettons. Après tout, ce n'est qu'une auto-proclamation...

II faut rappeler ici, pour mieux camper l'état de la société, la plus grande 'catastrophe' médiatique qu'ait connue l'Allemagne, et qui est associée au nom de la petite ville de Sebnitz en Saxe. Cette commune a fait l'expérience traumatique que, du jour au lendemain, quand un banal cliché semble devenir réalité, les médias sont capables de jeter aux orties tous leurs principes déontologiques de rigueur, 
de vérification de leurs sources et de véracité des faits. Le cliché : la sourde vision de la subsistance d'une Allemagne nazie à l'est. Certes, tous les commentateurs n'ont pas tiré de quelques faits inquiétants mais isolés observés dans quelques communes (surtout dans les régions mal desservies et au faible dynamisme économique) un constat selon lequel, foncièrement et généralement, l'Allemagne de l'est serait un repaire de réactionnaires xénophobes s'adonnant au rêve pangermanique. Mais il y eut (et il y a toujours, bien que moins prononcés) beaucoup de préjugés exprimant une tendance moralisatrice, se nourrissant à la fois de l'ignorance et du déni du réel, et culminant dans un préjugé collectif : celui d'une "Allemagne de l'ombre » à l'extrême-droite. C'est dans ce climat d'opinion qu'il n'était pourtant pas possible d'ignorer que, à la fin 2000 , même les publications et émissions les plus séreiuses renoncèrent à enquêter consciencieusement lorsque le quotidien de boulevard BILD, toujours en quête de sensationnel, rapporta que, dans le fond de l'Allemagne de l'est, à Sebnitz, des néonazis avaient, sous les regards de tous les témoins, noyé un enfant dans la piscine municipale.

Toujours est-il que, une fois prouvée la non véracité de cette allégation, cette 'catastrophe' médiatique géante a amené les médias de l'ouest à être plus prudents avec leurs apparentes certitudes et à se montrer plus critiques envers les clichés et schémas de pensée très prisés à l'ouest. Cela étant, il existera toujours des conjonctions thématiques favorables, y compris sous l'angle du 'politiquement correct'. Foncièrement, rien ne peut prémunir les médias contre la négligence, comme le révèle une affaire d'investigation reprise par la plupart des rédactions, et portant sur une collusion d'intérêts mêlant à Leipzig des personnalités en vue au milieu de la prostitution. Ici aussi, une fois la vérité rétablie, il apparut que le véritable scandale résidait dans l'amplification réciproque dans la course au sensationnel.

\section{Livre et cinéma : des vases communiquants}

Le marché du livre a connu une évolution moins heureuse car très vite, les puissants éditeurs de l'ouest ont pris une place prépondérante. Les maisons d'édition de l'est réputées comme Aufbau ou Volk und Welt ont perdu leurs bases. A l'inverse, un jeune auteur et journaliste est-allemand, Christoph Links, a mis toute son énergie dans la création des éditions du même nom publiant des livres à la couverture jaune canari. Son catalogue, qui joua un rôle important de forum lors de la quête identitaire des années de transition, a été systématiquement élargi en un lieu de réflexion sur les questions de politique et de société.

Un autre éditeur, profondément ancré dans la tradition de la République fédérale, Suhrkamp, a quitté Francfort/Main en 2009 pour s'installer à Berlin - un déménagement auquel les rubriques culturelles de la presse ont accordé une haute valeur symbolique, y voyant non sans raison le signe qu'émerge une nouvelle identité culturelle dans l'Allemagne unie. Pour qui sait à quel point cette maison d'édition était une institution représentative de la République de Bonn, combien elle a marqué des générations entières d'étudiants et d'intellectuels, la portée de ce déménagement s'impose d'elle-même. II s'agit de bien plus que d'une manifestation superficielle des migrations médiatiques ; il révèle que les lignes de force de la pensée sont en train de se réunir elles aussi tout naturellement préfigurant un milieu intellectuel allemand.

La même tendance s'observe quand on considère la Foire du livre de Leipzig qui est aujourd'hui une fête du livre réputée et appréciée, en complément du Salon du livre de Francfort, un forum largement plus marchand axé sur les stratégies commerciales et les ventes de licences. Ici non plus, personne ne distingue plus entre auteurs de l'est ou de l'ouest ni ne s'intéresse à d'éventuelles ou présumées différences culturelles. Et même si parfois, le curseur est placé sur de telles distinctions, cela ne change rien au fait qu'elles ont perdu tout leur sens. Un auteur est-allemand comme Thomas Brussig, maître dans l'art de l'ironie, est une star dans toute l'Allemagne, de même que Christa Wolf (qui fut longtemps une égérie
Principal risque aujourd'hui : le politiquement correct

De rares

nouveaux éditeurs à l'est

Un symbole de l'édition de l'ouest s'installe à l'est : Suhrkamp

Leipzig et Francfort : salons du livre allemand 
A la télévision des acteurs allemands qu'un cinéma, allemand de la RDA), non seulement s'exprime avec la sensibilité d'une femme avertie, mais est depuis longtemps déjà la Grande Cassandre de l'Allemagne unie.

Plus généralement encore, le monde de la création est étonnamment ouvert aux échanges, à l'enrichissement mutuel. Dans aucune chaîne, les directions des programmes en charge des œuvres de fiction ne peuvent plus concevoir de tourner un film sans de grands acteurs originaires de l'est comme Dagmar Manzel, Corinna Harfouch, Matthias Habich, Anja Kling, Jörg Schüttauf ou Winfried Glatzeder. Quant aux œuvres télévisuelles (allemandes tout court) de réalisateurs comme Andreas Kleinert (lauréat du Prix Adolf-Grimme) ou Andreas Dresen, elles sont assurées de se voir décerner les plus hautes distinctions comme les Prix Grimme (inventés à l'ouest). Ils avaient commencé très tôt à insuffler leurs manières de voir les choses, de même que la collection de fiction policière «Polizeiruf 110 » (quasi une 'marque' de la production de l'ex-RDA) fait aujourd'hui partie intégrante de l'identité du genre, développant le plus naturellement du monde des récits policiers réalistes et inscrits dans un cadre régional comme son grand concurrent d'origine ouest-allemande. Souvenons-nous : le premier épisode de «Tatort » collection classique et réputée de fictions policières (ouest à l'origine ; elle a été reprise en France sous le titre «Sur les lieux du crime »), diffusé il y a 40 ans, avait pour titre "Un taxi vers Leipzig » (Taxi nach Leipzig). Son propos était intra-allemand, tout comme celui de tant d'œuvres audiovisuelles aujourd'hui, de documentaires aussi - qu'ils traitent de la «République-théâtre » qu'était la RDA ou qu'ils soient consacrés à des sujets clés comme la voie de fuite des Allemands de l'Est qu'étaient les tunnels («Le tunnel »). Une preuve supplémentaire montrant que la télévision non seulement peut créer mais crée cet espace où s'épanouit la réflexion publique, celle de chaque individu comme de l'ensemble de la société.

Tout aussi remarquable est l'essor du cinéma, alors qu'on pensait que la liquidation de la société de production centrale de la RDA, la Defa, allait plonger la création dans la dépression. II faut dire que, lorsque le groupe Vivendi a pris position sur le vénérable site de production de la Defa (où résidait avant la guerre le géant européen Ufa) exclusivement pour y optimiser la rentabilité de ses activités immobilières (bureaux et habitations), rien ne laissait présager le renouveau de la production. Mais aujourd'hui, le site de Babelsberg est mondialement réputé. Et que dire de films comme "L'Allée du soleil », "Good Bye, Lenin! », "Un été à Berlin » ou «La vie des autres » ? Non seulement, ils ont apporté matière au dialogue intra-allemand - et même à ce qui est maintenant un 'monologue' allemand tout court -, mais ils concentrent désormais l'attention du monde entier auquel ils tendent le miroir du subtil processus de compréhension mutuelle des Allemands.

L'HISTOIRE DES MÉDIAS APRÈS L'UNITÉ ALLEMANDE est, malgré toutes ses ruptures et parfois ses occasions gâchées, dans l'ensemble une belle success story. Tout comme la réunification des deux Etats. Que ce soient les structures, les acteurs ou les vecteurs médiatiques, tous se sont révélés capables de créer un espace public favorable à la si difficile et si complexe reconstruction d'une identité qu'elle soit celle d'un individu, d'une région ou d'une nation. Le plus bel exemple de ce processus de multiples différenciations, rapprochements, assimilations, a été au printemps 2010 la candidature de Joachim Gauck à la fonction de président de la République fédérale. J. Gauck, un de ces opposants au régime de la RDA si représentatifs avec leurs liens avec l'Eglise protestante, et à qui le gouvernement fédéral confia plus tard la mission de gérer les archives et les dossiers de la Stasi, était considéré par les Allemands des milieux les plus divers comme la personnalité idéale pour représenter toute l'Allemagne. Et cette reconnaissance s'accompagnait d'un étonnant suivi par les médias, étonnant dans sa régularité comme dans celle de sa tonalité positive. Dans cette personnalité reconnue pour son intégrité absolue et au parcours digne du plus haut respect, on reconnaissait tout ce qui fait l'Unité de l'Allemagne.

Que cela ait pu se produire si naturellement et ait été ressenti comme allant de soi tient assurément beaucoup à la personnalité de J. Gauck. Mais cela tient bien 
plus - et bien plus essentiellement - encore au fait que l'Unité est largement réalisée "dans les têtes » des Allemands, par-delà toutes les différences régionales (qui existent entre l'est et l'ouest tout comme entre le nord et le sud). Or cela est - sans l'ombre d'un doute - le fruit d'une médiation médiatique qui, malgré tout ce qu'elle pouvait avoir de controversé ou de contradictoire, s'est toujours révélée foncièrement constructive et productrice de sens par le sérieux avec lequel elle abordait la profondeur des débats et l'intensité de sa confrontation avec la réalité des faits.

Traduction : I. Bourgeois

\section{Indications bibliographiques}

- « ARD im Gespräch. Eine andere Republik - ein anderer Rundfunk ? », débat diffusé par le Hessischer Rundfunk le 27 avril 2010

- BAHRMANN H., «Die organisierte Selbsttäuschung. DDR-Journalismus : eine Ausgrenzungsgeschichte. Anschlusspsychose und Personenwandlungen in Adlershof », epd / Kirche und Rundfunk, $\mathrm{n}^{\circ}$ 41, 26 mai 1990

- BouRgeols I., «Allemagne : une démocratie construite sur la libre circulation de l'information », Géopolitique, $\mathrm{n}^{\circ} 108$, janvier 2010

- BouRgeoIs I., «La télévision allemande, une indépendance structurelle », Le Temps des médias, $\mathrm{n}^{\circ} 13$, hiver 2009/2010

- Bourgeois I., « Comment la TV ouest-allemande a contribué à la chute du Mur. Un cas unique d'interaction entre médias et opinion », Working paper n¹9/1, 2009, www.cirac.u-cergy.fr

- DE Peretti B., «Témoignage. 9 novembre 1989. Conférence de presse à Berlin-Est », Working paper $n^{\circ} 19 / 2,2009$, www.cirac.u-cergy.fr

- «Deutschland - Heimat in Europa, 20 Jahre Wiedervereinigung », Die Welt, édition spéciale, 26 septembre 2010

- Dieckmann C., « Die Würde des Ostens. Was wir am 3. Oktober feiern können : Eine Bilanz nach zwanzig Jahren deutscher Einheit », Die Zeit, 30 septembre 2010

- « Es gab ein großes Vakuum‘ », interview de Roland Tichy, epd medien, n78, 6 octobre 2010

- « Goldene Zeiten. Wie manche die DDR-Medien verteilen wollen », epd / Kirche und Rundfunk, n¹2, 14 février 1990

- Hall P. C. (ed), « Revolutionäre Öffentlichkeit. Das Fernsehen und die Demokratisierung im Osten », Mainzer Tage der Fernsehkritik, vol. XXIII, Mayence, 1990

- Hochmuтh D., « Anschlusspsychose und Personenwandlungen in Adlershof », epd / Kirche und Rundfunk, $\mathrm{n}^{\circ} 41,26$ mai 1990

- «In Ruhe alle Kräfte zusammennehmen », interview de Gottfried Müller, ministre des médias de la RDA, epd / Kirche und Rundfunk, n47, 16 juin 1990

- « Konstruktive Konkurrenz. Gewerkschaftserklärung zur künftigen Rundfunkstruktur auf DDR-Gebiet », documenté in epd / Kirche und Rundfunk, n40, 23 mai 1990

- KammanN U., «Schnittmuster. Zur deutsch-deutschen Medienneuordnung », epd / Kirche und Rundfunk, $\mathrm{n}^{\circ} 41,26$ mai 1990

- Kloepfer I., Mrusek K., « Die unvollendete Einheit », Frankfurter Allgemeine Sonntagszeitung, 5 septembre 2010

- LOEST E., « Ost-West. Nord-Süd. Deutschland : Wie geeint ist die Nation nach 20 Jahren Einheit ? Ein subjektiver Blick auf das Land », Das Parlament, 6 septembre 2010

- MeYen M., « Die ARD in der DDR », Aus Politik und Zeitgeschichte, n²0, 17 mai 2010

- «Ministerrat: Beschluss zu statuarischen Grundsatzregelungen des Deutschen Fernsehfunks vom 15. März », documenté in epd / Kirche und Rundfunk, n²7, 7 avril 1990

- Petersen T., «Une nouvelle identité allemande? », Regards sur l'économie allemande, n91, mai 2009

- Prantl H., « Kain und Abel : Eine deutsche Geschichte », Süddeutsche Zeitung, supplément 20 Jahre deutsche Einheit, 2-3 octobre 2010

- SEIß G., «,Die Chance des Neuanfangs nutzen', Ziele einer Rundfunkentwicklung in der DDR », epd / Kirche und Rundfunk, n44/45, 9 juin 1990

- UlRich B., «Danke! 3. Oktober : Warum der Westen vom Osten pofitiert hat », Die Zeit, 30 septembre 2010

- «Umgang mit der Identität von Menschen », interview de Gero Hammer, epd / Kirche und Rundfunk, $n^{\circ} 44 / 45,9$ juin 1990

- «Unverwechselbar pro », interview de Wolfgang Vietze, directeur des programmes du Deutscher Fernsehfunk, epd / Kirche und Rundfunk, n²8/28, 11 avril 1990

- «Wer die Zuschauer hat, der wird gebraucht », interview de Hans Bentzien, Generalintendant du Deutscher Fensehfunk, epd / Kirche und Rundfunk, n²7, 7 avril 1990

- « 20 Jahre - 20 Geschichten », Berliner Zeitung, magazine spécial, 2-3 octobre 2010 


\section{Les médias à l'ère du numérique}

Réflexions franco-allemandes pour l'Europe

Sous la direction d'Isabelle Bourgeois

Travaux et documents du CIRAC Juillet 2008

ISBN 978-2-905518-36-1 160 p., $25 €$

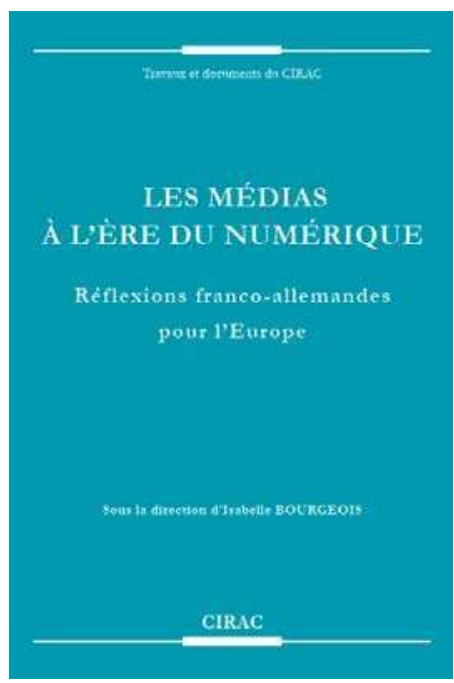

La télévision est en sursis. Bientôt, elle ne sera plus le seul média de référence : inexorablement, Internet poursuit sa montée en puissance, et le portable se métamorphose en téléviseur. La numérisation a brouillé les frontières entre les divers moyens de communication électroniques : audiovisuel et télécommunications convergent vers un nouvel ensemble aux contours indéfinis.

Comment l'Europe réagit-elle à ces mutations ? Comment en particulier la France et l'Allemagne abordent-elles ces défis ? Malgré d'importantes différences dans l'organisation concrète des marchés et de leur régulation, nos deux pays partagent foncièrement la même approche de la mission de l'audiovisuel, fondée sur la nécessité de garantir le pluralisme des opinions. Mais pourra-t-il encore assurer cette mission, indispensable au lien social, à l'ère du numérique ? Le consommateur des médias, le citoyen, est aujourd'hui largement libre de ses choix ; faut-il, et si oui, comment, le protéger contre les nouveaux risques susceptibles de naître dans un avenir médiatique encore incertain ? Face à la convergence de fonctions naguère séparées entre éditeurs, diffuseurs, fournisseurs d'accès, quelle approche faut-il développer pour veiller au libre jeu de la concurrence, au libre accès de tous au marché et à la libre circulation de l'information? Convient-il de soumettre à l'avenir les médias aux seules lois du marché ? Ou convient-il au contraire de développer un statut d'exception rénové ? Fautil, à l'ère du numérique, réguler plus, moins, ou autrement? Et, le cas échéant, où devrait se situer cette régulation : à l'échelon des Etats membres, à celui de l'Europe ?

Toutes ces interrogations nous renvoient à la question du sens que nous voulons donner au modèle de société qui naîtra des changements que traversent la France, l'Allemagne et l'Union européenne en ce début de l'ère du savoir et de la connaissance. Elles sont au coeur d'un dialogue entre journalistes spécialisés, experts, professionnels et chercheurs réputés, que retrace le présent ouvrage.

\section{SOMMAIRE}

\section{PRÉFACE de Victor Henle}

INTRODUCTION

Isabelle Bourgeois : La régulation entre deux logiques : prévoyance et libre concurrence

DÉBAT N 1 - Dans la jungle des nouveaux services. L'audiovisuel a-t-il vécu ?

(Francis Balle, Isabelle Bourgeois, Bernd Holznagel, Uwe Kammann, Bénédicte de Peretti)

DÉBAT $N^{\circ} 2$ - Nouveaux défis pour la régulation et le contrôle. Quels niveaux et quelles instances ? (Francis Balle, Dieter Dörr, Elisabeth Flüry-Hérard, Hans-Jürgen Jakobs, Jasmin Kundan)

DÉBAT N ${ }^{\circ} 3$ - Parts de capital ou parts d'audience. Ces critères sont-ils encore adaptés à l'ère du numérique ? (Isabelle Bourgeois, Jürgen Heinrich, Isabell Hülsen, Alain Lancelot, Dieter Schmidtchen)

DÉBAT N ${ }^{\circ} 4$ - Segmentation de l'offre, individualisation de la réception, atomisation de la société. Quelles bases de légitimité pour une régulation des médias ? (Jean-Louis Missika, Jacques Rigaud, Norbert Schneider, Andreas Stopp)

DÉBAT $N^{\circ} 5$ - Les entreprises s'européanisent. Faut-il un contrôle des concentrations pour les médias à l'écheIon européen ? (Olaf Christiansen, Peter Eberl, Thomas Kleist, Joseph Lorent, Werner Schwaderlapp)

POSTFACE de Victor Henle, Construire la démocratie de la communication

Ce Dialogue franco-allemand a été co-organisé à Berlin en novembre 2006 par la TLM, la LfM NRW et le CIRAC. Plus d'information et résumés des débats sur:

http://www.cirac.u-cergy.fr/debats.php\#media

http://www.tlm.de/tlm/aktuelles_service/veranstaltungen/archiv/2006/dialog/index.php 